\title{
Research on road engineering detection method based on GPR technology
}

\author{
Liu Tao ${ }^{1,2^{*}}$, Li Jia ${ }^{3}$, Zheng Zhi-gang ${ }^{2}$, Huang Zhi², Jiang Jian ${ }^{3}$, Hong Shao-you ${ }^{2}$, Li You-zhi ${ }^{3}$, and Wu Yin-tan ${ }^{2}$ \\ ${ }^{1}$ Shenzhen Tagen Engineering Technology Co., Ltd, 518034 Shenzhen, China \\ ${ }^{2}$ Shenzhen Yuetong Construction Engineering Co., Ltd, 518019 Ltd. Shenzhen, China \\ ${ }^{3}$ Shenzhen Tagen $<$ Group $>$ Co., Ltd, 518034 Shenzhen, China
}

\begin{abstract}
GPR is an effective non-destructive testing technology. This paper introduces its composition principle and operation method, explains the process of parameter setting and image optimization, obtains the dielectric constant of 10000 points, compares it with the density, and then obtains the uniformity distribution law of construction quality based on image. By calibrating the thickness of the road surface, the effective detection of road diseases can be realized, and the theoretical basis and practical application conditions of GPR technology can be clarified.
\end{abstract}

\section{Introduction}

The theoretical basis of GPR technology is to detect the underground medium by transmitting pulse electromagnetic wave. The medium with different dielectric constant will reflect the electromagnetic wave at the interface[1]. Due to the complex dielectric properties of underground media, different attenuation rates, complex and changeable geological conditions, it is difficult to measure the GPR. For road detection, the research focus is still focused on structural layer thickness detection, road void evaluation, disease detection and other fields[2]. And most of them can only obtain two-dimensional section information, the resolution is cm level, the time window is $0 \sim 2000 \mathrm{~ns}$, the center frequency is about $10 \sim 1000 \mathrm{MHz}$, and the detection depth is not more than $60 \mathrm{~m}$. The radar wave produced by the vibration source has good penetrating ability, wide bandwidth and high center frequency. It can also be used for the measurement and calculation of dielectric constant. This parameter is closely related to the construction quality indexes such as density and void ratio[3]. Through the introduction of 3D GPR system, this paper expounds the method of realizing high-quality radar data[4], so as to calculate the dielectric constant and provide a solid foundation for density prediction. At the same time, it introduces the reliable method of realizing road structural layer diseases and cavities under standard weather conditions[5], and verifies it with core drilling sampling, which provides a useful reference for the engineering community.

\section{Composition and operation method of 3D GPR system}

The three-dimensional GPR system adopted in this paper is composed of frequency stepping radar and antenna array as two important technical bases, which have high resolution ratio and are composed of host, antenna, laptop and other accessories. The so-called frequency step means that the working frequency of GPR is stepped in a step manner. In a scanning cycle, the transmitted signal rises from the starting frequency (f.start) to the ending frequency (f.stop) with the frequency interval $\Delta \mathrm{f}$, the working bandwidth $\mathrm{B}$ and the frequency interval $\Delta \mathrm{f}$ are $\mathrm{B}=(\mathrm{f}$.stop)-(f.start), $\Delta \mathrm{f}=\mathrm{B} /(\mathrm{N}-1)$ respectively. Among them, $\mathrm{N}$ is the number of stepped frequency points, and the digital frequency signal source is used to replace the traditional phase-locked loop. The system can generate a scanning cycle of $0.5-10 \mathrm{~ms}$, including 1,500 signals with different frequencies from $100 \mathrm{MHz}$ to $3 \mathrm{GHz}$. As a in-phase receiver, the whole signal can be fully utilized. Users can use different frequency weighting to reprocess the data, highlight the characteristics of interest, analyze the data in frequency domain, and reduce the external interference.

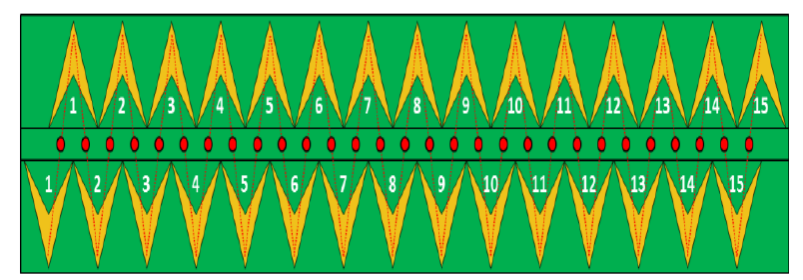

Fig. 1 Schematic diagram of internal vibrator arrangement of 3D GPR antenna

\footnotetext{
*Corresponding author: 315778102@qq.com
} 
Using the antenna array technology of air coupled butterfly unipole, there are 21 pairs of electronic scanning antenna arrays, which are arranged at a distance of $7.5 \mathrm{~cm}$, and 21 lines can be obtained by one acquisition, which improves the detection efficiency. Without replacing the antenna, the data of different detection depths can be obtained. At the same time, the characteristics of UWB technology is that there is a wide signal frequency band in the frequency domain, which can get more useful information and better echo response signals, and can improve the imaging accuracy of underground objects. UWB signal refers to the signal with $-10 \mathrm{~dB}$ greater than $500 \mathrm{MHz}$ or $-10 \mathrm{~dB}$ relative bandwidth greater than $20 \%$ of center frequency. The calculation formula is as follows:

The highest frequency is $\mathrm{f} \_\mathrm{H}$, the lowest frequency is $\mathrm{f} \_\mathrm{L}$, the relative bandwidth index of narrow-band signal is $\mu<1 \%$, the relative bandwidth index of wide-band signal is $1 \%-20 \%$, and the relative bandwidth index of ultra wideband signal is $\mu \geq 20 \%$.

$$
\mu=\frac{\Delta \mathrm{f}}{f_{o}}=2\left(f_{H}-f_{L}\right) /\left(f_{H}+f_{L}\right)
$$

During field testing, parameters such as the center frequency, sampling rate, time window length and scanning point spacing should be selected according to the situation. The scanning point spacing and antenna center frequency will significantly affect the detection effect, while the time window size and sampling rate will not have a significant impact on the data collection effect. In this paper, the frequency of three-dimensional GPR is $60 \mathrm{MHz}-2980 \mathrm{MHz}$, the frequency step is $20 \mathrm{MHz}$, the transmitting time of each frequency is 3 microseconds, the sampling time window is $25 \mathrm{~ns}$, and the longitudinal sampling interval is $26.3 \mathrm{~mm}$.

In a word, 3D GPR has good penetration ability, antijamming ability and target resolution ratio, and can realize multi-target detection and ultra-short range detection. By optimizing the signal bandwidth and setting the optimal resolution, it can achieve high-speed and large-scale scanning and exploration, and effectively avoid the loss of image details.

\section{Comparative researches on void ratio and dielectric constant of asphalt pavement}

This paper takes T22 standard asphalt pavement of Shantou Zhanjiang Expressway in Guangdong Province as the research object. SBS modified asphalt is used to test the penetration, penetration index, ductility $\left(5^{\circ} \mathrm{C}\right.$, $5 \mathrm{~cm} / \mathrm{min})$, softening point, kinematic viscosity $\left(135^{\circ} \mathrm{C}\right)$ and dynamic viscosity $\left(60^{\circ} \mathrm{C}\right)$. The coarse aggregate is clean, dry, hard, rough surface, full of edges and corners, the particle shape is close to the cube. The fine aggregate is clean, dry, free of weathering and impurities, and has appropriate grading. Through the test, the asphalt, coarse aggregate and fine aggregate meet the requirements of technical specifications. The gradation type of asphalt pavement's middle surface course is GAC-20C, the ratio of asphalt to stone is $4.5 \%$, the maximum theoretical relative density is $2.69 \mathrm{~g} / \mathrm{cm} 3$, and the designed void ratio is $4.3 \%$. The paving and rolling process are carried out according to the specification requirements.

In this paper, the PQI is used to measure the asphalt pavement, which has the characteristics of fast measurement speed, simple operation and good repeatability, and can display the density in real time. In order to verify the reliability of the method, three core drilling samples were selected and their densities were measured, which were $2.568 \mathrm{~g} / \mathrm{cm}^{3}, 2.644 \mathrm{~g} / \mathrm{cm}^{3}$ and $2.648 \mathrm{~g} / \mathrm{cm}^{3}$. The density measured by PQI is $2.673 \mathrm{~g} / \mathrm{cm}^{3}$, $2.618 \mathrm{~g} / \mathrm{cm}^{3}$ and $2.648 \mathrm{~g} / \mathrm{cm}^{3}$ respectively. It can be seen that there is little difference between the two, so it can be considered that the data measured by PQI is more reliable.

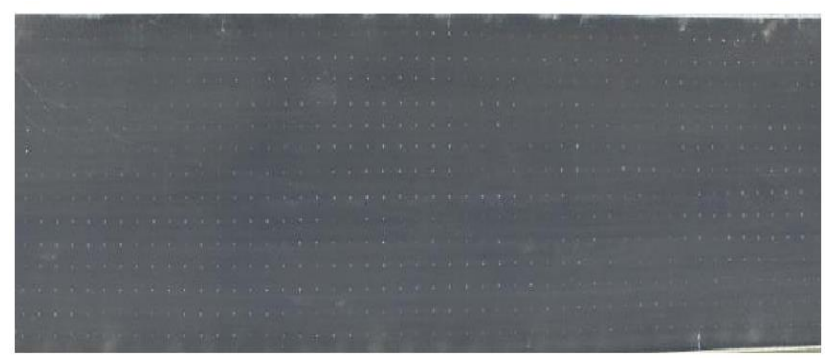

Fig. 2 Positioning of measuring points on the asphalt pavement

With this method, 10,000 points of density data are obtained. Since each point is next to four points, the average data of these five points can be taken as the representative value to obtain more accurate data. A grid with a distance of $1 \mathrm{~m}$ is drawn in the test road section with a tape measure, with a total length of $51 \mathrm{~m}$ and a total width of $13 \mathrm{~m}$. The outer edge of the grid is $1 \mathrm{~m}$ away from the road shoulder and the central reservation. After calculation, the proportion of $0 \%-3 \%$ void ratio is $32 \%$, the proportion of $3 \%-6 \%$ void ratio is $62.9 \%$, the proportion of $6 \%-9 \%$ void ratio is $5.1 \%$. Using MATLAB software, the corresponding program is compiled to draw the three-dimensional distribution diagram of the void ratio of asphalt pavement. The smaller the void ratio is, the lower the position is, the bluer the color is, the larger the void ratio is, the higher the position is, and the redder the color is.

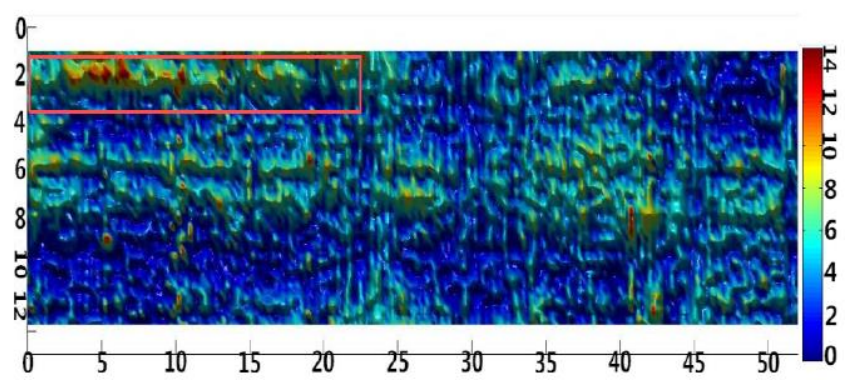

Fig. 3 Void distribution of asphalt pavement

From figure 3 it can be clearly seen that the distribution condition of void ratio of asphalt pavement. The void ratio of red box area is large, and there is obvious segregation. In fact, it is the overlapping position of paver, and the void ratio is obviously large. 
The dielectric constant is measured by the method of common midpoint. For the purpose of comparative analysis, it is consistent with the measurement point of the PQI and the corresponding data are obtained. The basic principle of the common midpoint method is to calculate the dielectric constant of the medium by obtaining the propagation time and distance of the radar wave at different measuring angles, which has a strict theoretical basis.

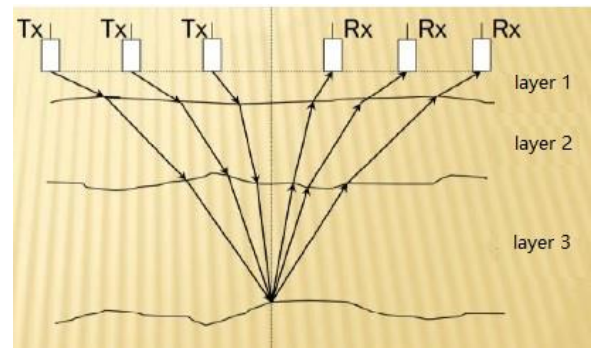

Fig. 4 Common midpoint measurement method of 3D GPR

Through the automatic tracking radar wave reflection interface, the basis of calculation based on the common midpoint method is obtained, the program is compiled, and the automatic calculation is realized, as shown in Figure 4. The 3D-Radar Examiner software is used to process the corresponding measurement source file of common midpoint. The attenuation of ISDFT item is set to 0.04 , the start depth (ns) of BGR (high pass) item is set to 2.7, and then click the Process Selected Regions item to process and save to the corresponding file. The application method of the program is: click to create a new project, the path of the source file is the location saved after the above steps, import the file, analysis distance is $0.25 \mathrm{~m}$, and click to select the file to calculate automatically.

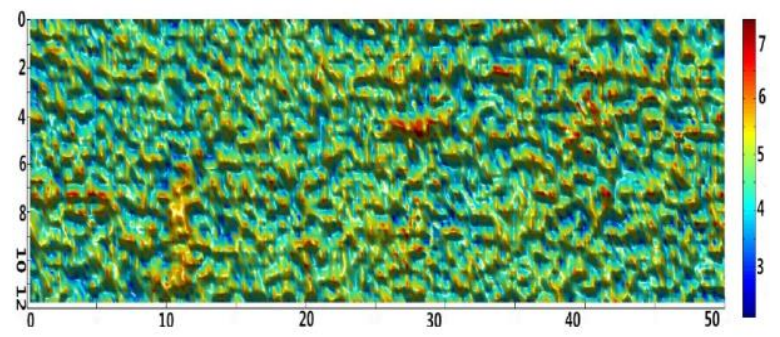

Fig. 5 Dielectric constant distribution of asphalt pavement

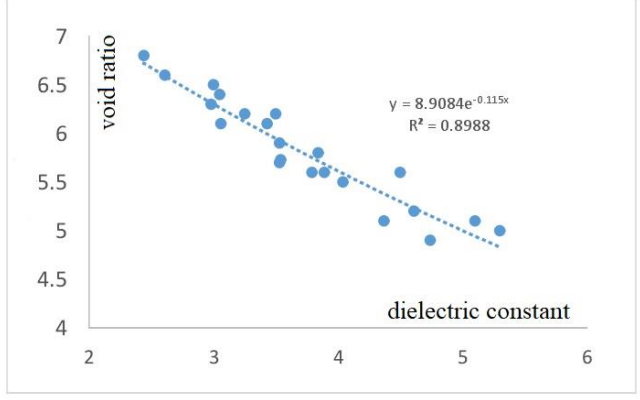

Fig. 6 Correlation between void ratio and dielectric constant of asphalt pavement

In the same way, MATLAB software is used to draw the three-dimensional dielectric constant distribution map of asphalt pavement. The smaller the dielectric constant is, the lower the position is, the bluer the color is; the larger the dielectric constant is, the higher the position is, and the redder the color is. By comparing Fig. 3 and Fig. 5, we can see that there is no obvious correlation between them. This is because there is an exponential correlation between dielectric constant and void ratio, so it is not possible to directly compare the two pictures.

By selecting the data of dielectric constant (common midpoint method) and density (PQI method) of 30 points, the fitting curve is established. It can be seen that the larger the dielectric constant is, the smaller the void ratio is, the smaller the dielectric constant is, and the larger the void ratio is, which conforms to the electromagnetic mixing theory. The above method is feasible.

\section{Application of GPR technology in road structure detection}

Using 3D GPR software for data processing is to obtain the dielectric constant of asphalt pavement based on experience or core drilling sampling, i.e. assuming that the value is a fixed value, in fact, the dielectric constant is variable. In other words, using the constant dielectric constant to calculate the thickness of asphalt pavement is not rigorous in theory and may have errors. In this paper, the dielectric constant of each point or section (certain scale) of asphalt pavement is calculated and substituted into formula $d=c t /(2 \sqrt{\varepsilon})$ to modify the thickness of asphalt pavement[6]. On the other hand, Time Grounds used in the software is a fixed value, and the value is variable. The nanosecond change of radar wave propagation time in asphalt pavement may cause a large change of dielectric constant.

Table 1. Dielectric constant of asphalt pavement based on GPR technology

\begin{tabular}{|c|c|c|c|c|}
\hline Station & $\begin{array}{c}\mathrm{t} 1 \\
(\mathrm{~ns})\end{array}$ & $\begin{array}{c}\mathrm{t} 2 \\
(\mathrm{~ns})\end{array}$ & $\begin{array}{c}\text { thickness } \\
(\mathrm{m})\end{array}$ & $\begin{array}{c}\text { Dielectric } \\
\text { constant }\end{array}$ \\
\hline $\mathrm{K} 2115+800$ & 2.93 & 6.35 & 0.185 & 7.69 \\
\hline $\mathrm{K} 2115+390$ & 3.03 & 6.05 & 0.19 & 5.7 \\
\hline $\mathrm{K} 2114+900$ & 2.93 & 5.86 & 0.175 & 6.3 \\
\hline $\mathrm{K} 2093+800$ & 2.82 & 5.02 & 0.108 & 9.36 \\
\hline $\mathrm{K} 2094+000$ & 2.82 & 4.95 & 0.122 & 6.86 \\
\hline
\end{tabular}

Taking the K2114+900 sample of Beijing Zhuhai Expressway as an example, the two-way propagation time t1 of radar wave air-road surface is $2.93 \mathrm{~ns}$, and the two-way propagation time $\mathrm{t} 2$ of radar wave-road bottom is $5.86 \mathrm{~ns}$, so the dielectric constant is 6.3 . If $\mathrm{t} 1$ is $3.03 \mathrm{~ns}$ and other parameters remain unchanged, then the dielectric constant is 5.884 , with a difference of 0.416 . It can be seen that by tracking the interface line of the airroad surface, a program is written to automatically identify the first peak value of the amplitude, which can accurately output $\mathrm{t} 1$. 


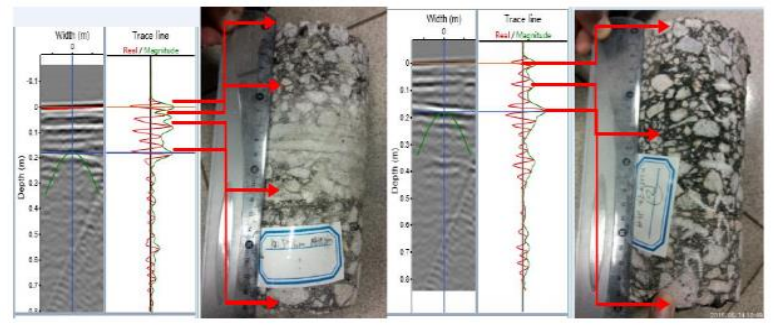

(1) $\mathrm{K} 2115+800$

(2) $\mathrm{K} 2115+390$

Fig. 7 Calculation of asphalt pavement thin layer thickness based on GPR technology

It can be seen from the above figure that the threedimensional GPR can well identify the upper, middle and lower layers of asphalt pavement, and the interface is relatively clear. This is because the materials used in the three layers are different, and their dielectric constants are also different. It is worth noting that radar image is only the basis for intuitive judgment, and the radar wave information of asphalt pavement foundation that can generate image is the key to data processing and thickness calculation, and special attention should be paid to the analysis and interpretation of radar wave trace line (Trace line).

Generally, the cracking of asphalt pavement structure is mainly vertical fracture, with few inclined cracks, especially the structural damage cracks caused by driving load. The mechanism is that when the strength of wheel load is greater than the tensile strength of semirigid base, the bottom of semi-rigid base will crack quickly, and with the increase of load action times, the bottom cracks will gradually expand to the upper part. The radar image in Figure 8 clearly reflects the crack area including asphalt surface course, base course and top of subgrade, so overhaul is required.

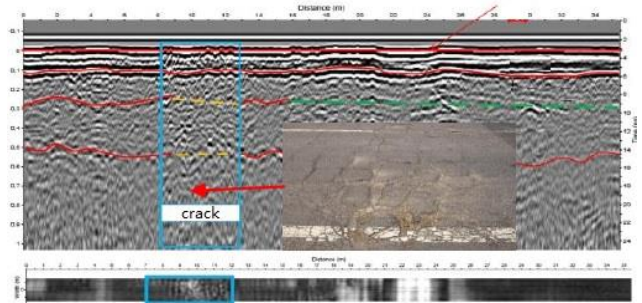

Fig. 8 Crack area

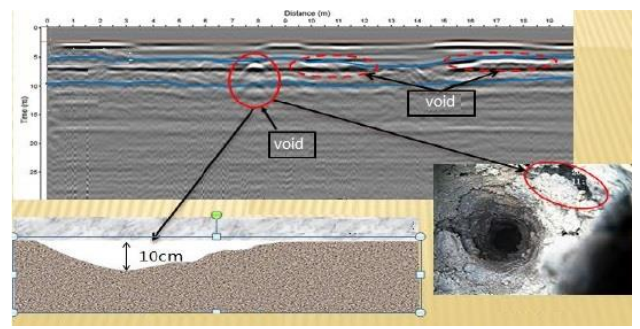

Fig. 9 Void area

The reflection time of mine detection wave at each interface of the pavement structure layer is relatively short. Part of the energy of the incident wave is reflected at the top of the base layer, and the other part continues to project downward. If the interface contacts well and there are no abnormality, the incident wave will penetrate deeper, generally without obvious reflection. However, if the asphalt pavement is in poor contact with the base course and there are voids, there will be an obvious air layer, and the radar wave will have a time mutation, with a large waveform change. As shown in Figure 9, there will be a significant radar wave reflection, which has been proved to be a large void through field core drilling sampling.

\section{Conclusions}

This paper introduces various methods of GPR used in road engineering detection, including composition principle and operation method, relationship between dielectric constant and density of asphalt pavement, road thickness and cavity detection, etc. The conclusions are as follows:

(1) Three dimensional GPR technology is based on frequency stepping and antenna array. It can provide high-definition, large-scale GPR images and data, and provide convenient conditions for dielectric constant calculation, cavity detection and other tasks.

(2) Through the measurement of 10,000 points in the asphalt pavement, the data of void ratio and dielectric constant are obtained, but only in terms of threedimensional images, they are not well correlated. This is because they are exponential relations. Through the data fitting tool, the relationship between porosity and density can be defined, which is helpful to broaden the application boundary of GPR.

(3) Using fixed dielectric constant and air-road surface interface line will affect the detection of asphalt pavement thickness by GPR. By adjusting the frequency and setting parameters, 3D GPR can identify the thin layer of asphalt pavement and the cavity of road structure.

\section{References}

1. Olhoeft G R. Applications and frustrations in using ground penetrating radar[J]. IEEE aerospace and electronic systems magazine, 2002, 17(2): 12-20.

2. $\mathrm{Xu}$ Sheng-cai, Liu Feng. The Research and Application of GPR to Detect Thickness in City Road [D]., 2000.

3. Ameri M, Kashani Novin M, Yousefi B. Comparison of the field measurements of asphalt concrete densities obtained by ground-penetrating radar, pavement quality indicator and the borehole coring methods[J]. Road Materials and Pavement Design, 2014, 15(4): 759-773.

4. Deng Shikun. On The Basic Principles for the choice of GPR field survey parameters [D], 2005.

5. LU Cheng-Ming, QIN Zhen, ZHU Hai-Long, Practical methods for detection of concealed cracks in highway pavement using ground penetration radar dat. Chinese journal of Geophysics 2007, 50(5): 1558-1568. 
6. 2008 J. Regulations for field test of highway subgrade and pavement [S][D]., 2008. 